\title{
47
}

\section{Schlangenrätsel 6}

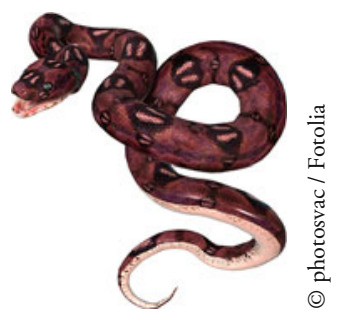

Im folgenden Buchstabenfeld hat sich wieder ein weiser Spruch eingeschlängelt. Der Anfangsbuchstabe (S), der Endbuchstabe (R), sowie zwei ganze Wörter (KATER, ER) sind grau markiert. Umlaute werden umgewandelt zu $\mathrm{UE}, \mathrm{AE}$ und $\mathrm{OE}$. Findest du die Wortschlange?

\begin{tabular}{|c|c|c|c|c|c|c|c|c|c|c|c|c|}
\hline$E$ & $N$ & $E$ & $\mathrm{~T}$ & $\mathrm{~L}$ & $E$ & $F$ & $\mathrm{G}$ & $P$ & I & $\mathrm{J}$ & $\mathrm{H}$ & $S$ \\
\hline$R$ & Y & $\mathrm{T}$ & $\mathrm{M}$ & K & $S$ & $L$ & $\mathrm{M}$ & $D$ & $\mathrm{P}$ & I & W & $\mathrm{R}$ \\
\hline$N$ & $\mathrm{~L}$ & $\mathrm{G}$ & D & B & Z & $N$ & $A$ & $C$ & r & $A$ & $U$ & $E$ \\
\hline U & I & L & $\mathrm{J}$ & r & I & K & K & 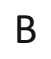 & $U$ & $\Gamma$ & 0 & $\mathrm{~T}$ \\
\hline $\mathrm{R}$ & V & $A$ & $\mathrm{~T}$ & $E$ & $\mathrm{R}$ & $\mathrm{N}$ & $\mathrm{M}$ & I & $\mathrm{J}$ & $\mathrm{K}$ & & $A$ \\
\hline $\mathrm{N}$ & K & $\mathrm{G}$ & D & Z & 0 & V & G & $A$ & $Q$ & V & $\mathrm{H}$ & $\mathrm{K}$ \\
\hline$A$ & $\mathrm{H}$ & $N$ & $\mathrm{~N}$ & $A$ & C & $\mathrm{H}$ & $\mathrm{T}$ & $\mathrm{s}$ & $B$ & $P$ & & $\mathrm{~N}$ \\
\hline $\mathrm{H}$ & I & K & $\mathrm{M}$ & I & $P$ & $N$ & $\mathrm{H}$ & A & $U$ & $F$ & $D$ & $E$ \\
\hline $\mathrm{R}$ & $E$ & $D$ & $\mathrm{~T}$ & $\mathrm{H}$ & $\mathrm{R}$ & $F$ & 1 & & $X$ & $A$ & $D$ & $N$ \\
\hline 0 & $D$ & K & G & $U$ & $\mathrm{M}$ & $M$ & $Y$ & $\mathrm{~N}$ & G & I & $\mathrm{T}$ & $U$ \\
\hline$S$ & $\mathrm{~T}$ & $E$ & 1 & $\mathrm{~T}$ & $P$ & 1 & K & 3 & D & $F$ & $\mathrm{~J}$ & $\mathrm{M}$ \\
\hline
\end{tabular}

\section{Lösung:}

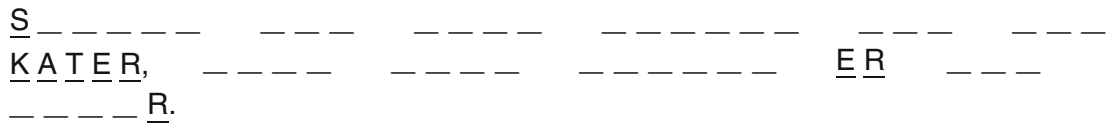

(C) Springer-Verlag Berlin Heidelberg 2016 